\title{
Efeito da Temperatura Ambiente e do Sistema de Criação sobre as Exigências de Energia Metabolizável para Mantença de Aves Reprodutoras Pesadas ${ }^{1}$
}

\author{
Carlos Bôa-Viagem Rabello², Nilva Kazue Sakomura ${ }^{3}$, Flavio Alves Longo ${ }^{3}$, \\ Kleber Tomas de Resende ${ }^{3}$
}

\begin{abstract}
RESUMO - O trabalho foi conduzido com a finalidade de verificar o efeito da temperatura e do sistema de criação sobre as exigências de energia metabolizável aparente para mantença (EMm) para aves reprodutoras pesadas. No experimento 1, foram determinadas as exigências de EMm para aves criadas em gaiolas. Foram alojadas 192 aves reprodutoras pesadas, Hubbard HI-Y em galpões com temperaturas controladas a 13,21 e $30^{\circ} \mathrm{C}$. As aves foram distribuídas de acordo com delineamento inteiramente casualizado, com quatro tratamentos e quatro repetições de quatro aves. Os tratamentos foram: consumo de ração ad libitum, 70,50 e $30 \%$ em relação ao consumo ad libitum. Quantificou-se a energia retida (ER) pela técnica do abate comparativo. A partir das análises de regressão da energia metabolizável ingerida (EMI), em função da ER corporal, determinaram-se as exigências de EMm e as eficiências de utilização da energia ( $k$ ). No experimento 2, as exigências de EMm foram determinadas para aves criadas em piso. Foram distribuídas 96 aves em um delineamento experimental casualizado, em três temperaturas $\left(13,21\right.$ e $\left.30^{\circ} \mathrm{C}\right)$ e quatro repetições de oito aves. Quantificou-se a ER pelo abate comparativo e a EMm, pela fórmula EMm=EMI-(ER/k). As exigências de EMm para aves criadas em gaiolas foram: 111.2, 91.5 e $88,5 \mathrm{kcal} / \mathrm{kg}^{0,75} / \mathrm{dia}$ e para as aves criadas em piso, $130.8,112.9$ e $111.0 \mathrm{kcal} / \mathrm{kg}^{0,75} / \mathrm{dia}$ para as temperaturas de 13,21 e $30^{\circ} \mathrm{C}$, respectivamente. As exigências das aves criadas em piso foram, em média, 21,8\% superiores às alojadas em gaiolas, provavelmente devido aos maiores gastos de energia com atividade física.
\end{abstract}

Palavras-chave: aves reprodutoras pesadas, energia metabolizável para mantença, sistema de criação, temperatura ambiente

\section{Effect of the Environmental Temperature and Rearing Systems on Metabolizable Energy Requirements for Maintenance of Broiler Breeders Hens}

\begin{abstract}
This study was conducted to evaluate the effect of the temperature and rearing system on the apparent metabolizable energy requirements for maintenance (MEm) for broiler breeder hens. In the experiment 1, MEm was determined with birds in cages. One hundred-ninety-two birds were housed in climatic chambers at 13, 21 and $30^{\circ} \mathrm{C}$. The birds were assigned to four treatments with four replications of four birds each. The treatments were: ad libitum intake, 70, 50 and 30\% of ad libitum intake. Comparative slaughter technique was used to determine the retained energy (RE). Maintenance metabolizable energy requirements (MEm) and efficiency of energy utilization $(k)$ were determined by the ME intake (MEI) and retained energy (RE) ratio. In the experiment 2, the MEm was determined with birds on the floor. Ninety-six birds were allotted to three temperatures $\left(13,21\right.$ e $\left.30^{\circ} \mathrm{C}\right)$ with four replication of eight birds each. The comparative slaughter technique was used to determined the RE and MEm requirements was determined by: EMm=EMI(RE/k). The MEm requirements for birds in cages were $111.2,91.5$ and $88.5 \mathrm{kcal} / \mathrm{kg}^{0.75} / \mathrm{day}$ and for birds on floor $130.8,112.9$ and $111.0 \mathrm{kcal} / \mathrm{kg}^{0.75} /$ day at 13,21 and $30^{\circ} \mathrm{C}$, respectively. Birds raised on floor had MEm higher $21.8 \%$ than those in cages, probably due to the energy expenditure for physical activity.
\end{abstract}

Keys Words: broiler breeder hens, environmental temperature, maintenance energy requirements, reading system

\section{Introdução}

A exigência de energia para mantença pode ser definida como aquela necessária para o metabolismo basal, para a produção de calor e para atividades normais, estando diretamente relacionada ao peso corporal e à temperatura (Grimbergen, 1974).
Chwalibog (1985) e Macleod \& Jewitt (1988) comentam que $65 \%$ da energia metabolizável ingerida pelas aves é perdida como calor e apenas $35 \%$ está disponível para produção.

Dados sobre as exigências de energia metabolizável para mantença de aves reprodutoras pesadas são bastante escassos. Estudos com o obje-

\footnotetext{
1 Parte da Tese de Doutorado do primeiro autor, apresentada à FCAV-UNESP-Jaboticabal, São Paulo. Pesquisa financiada pela FAPESP. ${ }^{2}$ Departamento de Zootecnia, Universidade Federal Rural de Pernambuco - UFRPE, Rua Dom Manoel de Medeiros, s/n, Dois Irmãos, Recife, PE, CEP: 52171-900 (cbviagem@ufrpe.br).

${ }^{3}$ Departamento de Zootecnia, Faculdade de Ciências Agrárias e Veterinárias, UNESP, Via de Acesso Prof. Paulo D. Castellane, s/n, Jaboticabal, São Paulo, CEP: 14.870-000 (sakomura@ fcav.unesp.br).
} 
tivo de determinar estas exigências foram realizados por Jonhson \& Farrell (1983) e Spratt et al. (1990), que encontraram valores similares de 87,24 e $87,71 \mathrm{kcal} / \mathrm{kg}^{0,75} / \mathrm{dia}$, respectivamente, quando alojaram reprodutoras pesadas em câmara de respiração à temperatura de $21^{\circ} \mathrm{C}$.

Por outro lado, alguns pesquisadores revelam que existem diversos fatores que influenciam as exigências de energia para mantença das aves, destacando-se a temperatura ambiente e o grau de atividade do animal. Os efeitos da temperatura sobre as exigências de energia metabolizável para mantença de aves têm sido estudados por diversos pesquisadores e a maior parte dos trabalhos revela efeito linear da temperatura sobre as exigências de mantença das aves (Rabello, 2001). No entanto, Peguri \& Coon (2000) demonstraram efeito quadrático quando estudaram poedeiras leves submetidas a diferentes temperaturas. Entretanto, segundo Hurwitz et al. (1980), o efeito da temperatura no metabolismo energético é muito complexo o que muitas vezes levam a respostas cúbicas, e não lineares, como geralmente se preconiza.

Segundo Austic \& Nesheim (1990), as exigências de energia para atividade são consideravelmente variáveis e, normalmente, estimadas em aproximadamente $50 \%$ do metabolismo basal, sendo, no entanto, influenciadas pelas condições de alojamento. Em aves poedeiras, aproximadamente 20 a $25 \%$ da produção diária de calor são decorrentes sa atividade (Macleod et al., 1982; Boshouwers \& Nicaise, 1983, 1985). Li et al. (1991) também relatam que em torno de $19 \%$ da energia gasta com a produção de calor é devido à atividade e, em condições de luz e escuro, a diferença na produção de calor foi de $33 \%$ em aves poedeiras. Todavia, outros tipos de atividades tem efeitos variáveis na perda de calor e diferenças no temperamento e, portanto, o genótipo, plano de nutrição, grau de empenamento, além da temperatura ambiente, são fatores importantes na determinação da perda de calor por atividade (Balnave et al., 1974).

Assim, o objetivo deste trabalho foi verificar os efeitos da temperatura e do sistema de criação sobre as exigências de energia metabolizável para mantença de aves reprodutoras pesadas na fase de produção.

\section{Material e Métodos}

Foram conduzidos dois experimentos no Setor de Avicultura do Departamento de Zootecnia da Faculdade de Ciências Agrárias e Veterinárias, Campus de Jaboticabal - UNESP. Os experimentos foram realizados com reprodutoras pesadas, Hubbard HI-Y, com 29 semanas de idade, alojadas em três câmaras climatizadas com temperaturas controladas de 13,21 e $30^{\circ} \mathrm{C}$.

Os experimentos foram conduzidos durante seis semanas, sendo uma de adaptação das aves e cinco de período experimental. Para determinação das exigências de energia metabolizável para mantença (EMm), utilizou-se a técnica do abate comparativo quantificando-se a retenção da energia consumida durante o período experimental por meio da determinação da energia contida na carcaça das aves no início dos experimentos e a energia retida na carcaça e ovos pelas aves até o final. Foram abatidas 4 aves/ temperatura no início do experimento e todas as aves no final.

\section{Experimento 1}

No experimento 1 , foram determinadas as exigências de energia metabolizável de mantença (EMm) para aves criadas em gaiolas. Foram utilizadas 192 aves, sendo 64 aves em cada temperatura, alojadas em gaiolas $(100 \times 45 \times 40)$ de acordo com delineamento experimental inteiramente casualizado (DIC). Os tratamentos consistiram de quatro níveis de ingestão de alimento (ad libitum, 70, 50 e 30\% em relação ao consumo ad libitum), com quatro repetições de 4 aves/gaiola em cada uma das temperaturas. As aves receberam ração balanceada de acordo com as exigências nutricionais, conforme recomendações do manual da linhagem (Tabela 1).

A ingestão de energia metabolizável (EMI) foi estimada a partir da quantificação da ração ingerida no período. A energia retida total (ER) foi estimada pela análise da composição corporal e dos ovos (energia final retida menos energia inicial retida), sendo por diferença, determinada a produção de calor (PC) por intermédio da seguinte fórmula $\mathrm{PC}=$ EMI-ER, em $\mathrm{kcal} / \mathrm{kg}^{0,75} / \mathrm{dia}$.

As variáveis ingestão de energia, energia retida e produção de calor foram relacionadas por meio de 
equações de regressão, de acordo com o proposto por Farrel (1974), para obtenção das exigências diárias de energia metabolizável para mantença (EMm) e a eficiência de utilização da EM da dieta foi calculada conforme Lofgreen \& Garret (1968) para determinar a produção de calor do jejum, isto é, a exigência de energia líquida para mantença.

\section{Experimento 2}

No experimento 2, foram determinadas as exigências de EMm para aves criadas em piso. Foram utilizadas 96 aves da linhagem Hubbard HI-Y, alojadas em boxes de 1,00 x 3,00 m. As aves foram distribuídas em delineamento inteiramente casualizado, com três temperaturas $\left(13,21\right.$ e $\left.30^{\circ} \mathrm{C}\right)$ e quatro repetições de oito aves. As quantidades de ração foram fornecidas (Tabela 1), conforme recomendação do manual da linhagem (Hubbard Farms, 2000). As exigências de EMm foram determinadas pela seguinte formula: $\mathrm{EMm}=\mathrm{EMI}-(\mathrm{ER} / k)$, em que $k=$ eficiência de utilização da energia para retenção no corpo e ovos foi determinada no experimento 1 .

Abate das aves e processamento das carcaças, penas e ovos

As aves foram submetidas a jejum de 24 horas, previamente ao sacrifício por deslocamento cervical, sendo em seguida pesadas individualmente, escaldadas, depenadas automaticamente e congeladas. Uma amostra contendo cerca de $100 \mathrm{~g}$ de penas foi armazenada. Em seguida, as carcaças foram serradas, moídas com posterior coleta de amostras e secagem em estufa a $55^{\circ} \mathrm{C}$ por 72 horas. As amostras de penas foram finalmente cortadas e as amostras de ovos coletadas no início e final dos experimentos foram homogeneizadas em liqüidificador por 2 minutos, sendo depois secas em estufa nas mesmas condições das amostras de carcaça.

\section{Análises laboratoriais}

As análises de energia bruta das amostras de carcaças, penas e ovos, coletadas durante os experimentos foram realizadas em bomba calorimétrica, segundo metodologia descrita pela AOAC (1990).

\section{Análises estatísticas}

Para determinar o efeito da temperatura sobre as exigências de energia metabolizável dos dois experimentos, fez-se a regressão dos valores de EMm determinados em cada temperatura, em função da temperatura ambiente pelo procedimento PROC REG do programa computacional SAS (SAS, 1996).

\section{Resultados e Discussão}

Na Tabela 2, são apresentados os dados de ingestão de energia, retenção de energia corporal e produção de calor das aves alojadas em gaiolas submetidas a diferentes temperatura e níveis de alimentação. Os maiores consumos nas temperaturas mais baixas refletiram em maiores exigências de energia para mantença, enquanto as aves submetidas a temperaturas acima da zona de termoneutralidade $\left(30^{\circ} \mathrm{C}\right)$ tiveram redução no consumo alimentar pela necessidade em dissipar calor, refletindo, conseqüentemente em menor deposição de energia. Segundo Rutz (1994), quando a temperatura ambiental aumenta, a ave reduz seu consumo, na tentativa de manter sua temperatura dentro dos limites homeostáticos.

Tabela 1 - Composição percentual da ração experimental Table 1 - Percentage composition of the experimental diet

\begin{tabular}{|c|c|}
\hline \multirow[t]{2}{*}{$\begin{array}{l}\text { Ingrediente } \\
\text { Ingredients }\end{array}$} & $\begin{array}{c}\text { Composição da ração } \\
\text { Ration composition }\end{array}$ \\
\hline & $(\%)$ \\
\hline Milho & 68,670 \\
\hline Corn & \\
\hline Farelo de soja & 20,991 \\
\hline Soybean meal & \\
\hline Fosfato bicálcico & 1,840 \\
\hline Dicalcium phosphate & \\
\hline Calcário & 7,558 \\
\hline Limestone & \\
\hline Sal comum & 0,350 \\
\hline Salt & \\
\hline DL-Metionina & 0,191 \\
\hline DL-methionine & \\
\hline Suplemento vitamínico & 0,300 \\
\hline Vitamin premix & \\
\hline $\begin{array}{l}\text { Suplemento mineral } \\
\text { Mineral premix }\end{array}$ & 0,100 \\
\hline Composição calculada & \\
\hline Calculated composition & - \\
\hline Proteína bruta, $\%$ & 15,500 \\
\hline Crude protein & \\
\hline Energia metabolizável, kcal/kg & 2.825 \\
\hline Metabolizable energy & \\
\hline Metionina, \% & 0,446 \\
\hline Methionine & \\
\hline Metionina+Cistina, \% & 0,715 \\
\hline Methionine + cystine & \\
\hline Lisina, \% & 0,760 \\
\hline Lysine & \\
\hline Cálcio, \% & 3,390 \\
\hline Calcium & \\
\hline Fósforo disponível, \% & 0,410 \\
\hline Available phosphorus & \\
\hline
\end{tabular}


As aves que receberam níveis de restrição mais severos em todas as temperaturas apresentaram retenções de energia negativas, em razão da mobilização de reservas corporais para atender as exigências de mantença (Tabela 2).

As equações de regressão para retenção de energia corporal em função da energia metabolizável ingerida e para produção de calor geraram os valores de EMm, ELm, e eficiências de utilização da energia (k) nas diferentes temperaturas $\left(13,21\right.$ e $\left.30^{\circ} \mathrm{C}\right)$ são apresentadas na Tabela 3. As exigências de EMm e ELm foram superiores nas temperaturas mais baixas, devido à demanda de energia para produção de calor e manutenção da homeotermia.

Os valores de energia líquida para mantença (ELm) representados pela produção de calor em jejum das aves, foram 77,$8 ; 65,2$ e $59,2 \mathrm{kcal} / \mathrm{kg}^{0,75} / \mathrm{dia}$ nas temperaturas de 13,21 e $30^{\circ} \mathrm{C}$, respectivamente (Tabela 3). Estes resultados foram inferiores aos encontrados por Basaglia (1999), para poedeiras leves alojadas em gaiolas submetidas à temperaturas similares $\left(12,22\right.$ e $\left.31^{\circ} \mathrm{C}\right)$, que obteve valores de 100,0 ; 79,$9 ;$ e $69,2 \mathrm{kcal} / \mathrm{kg}^{0,75} / \mathrm{dia}$, respectivamente. Marsden $\&$ Morris (1987) encontraram valores maiores na

Tabela 2 - Índice relativo (IR) da ingestão de energia metabolizável aparente (EMAn), retenção de energia corporal e produção de calor de reprodutoras pesadas criadas em gaiolas submetidas à temperaturas de 13,21 e $30^{\circ} \mathrm{C}$

Table 2 - Relative value (IR) of ME intake, body retained energy and heat production (HP), $\mathrm{kcal} / \mathrm{kg} .75 / \mathrm{day}$, for broiler breeder hens reared in cages and under 13,21 and $30^{\circ} \mathrm{C}$

\begin{tabular}{|c|c|c|c|c|}
\hline \multirow{2}{*}{$\begin{array}{l}\text { Nível de ingestão } \\
\text { Intake level }\end{array}$} & & \multicolumn{3}{|c|}{$\begin{array}{c}\text { Temperatura }\left(13^{\circ} \mathrm{C}\right) \\
\text { Temperature }\end{array}$} \\
\hline & $\begin{array}{l}\mathrm{IR}^{2} \\
(\%)\end{array}$ & $\begin{array}{c}\text { Ingestão de EMAn } \\
\text { (kcal/kg0,75/dia) } \\
\text { ME intake } \\
\left(\text { kcal } \mathrm{kg}^{7}{ }^{75} / \text { day }\right)\end{array}$ & 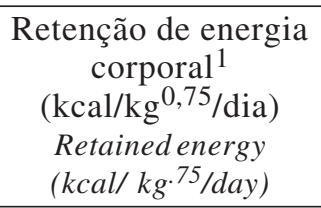 & $\begin{array}{c}\text { Produção de calor } \\
\text { (kcal/kg0,75/dia) } \\
\text { Heat production } \\
\text { (kcal/ } \mathrm{kg}^{75} / \text { day) }\end{array}$ \\
\hline $\mathrm{T} 1$ - ad libitum & 100,0 & 216,68 & 61,77 & 154,91 \\
\hline $\mathrm{T} 2-70 \%$ & 72,2 & 156,42 & 29,99 & 126,43 \\
\hline $\mathrm{T} 3-50 \%$ & 51,4 & 111,32 & 4,57 & 106,74 \\
\hline $\mathrm{T} 4-30 \%$ & 32,2 & 69,69 & $-29,65$ & 99,33 \\
\hline \multirow{2}{*}{$\begin{array}{l}\text { Nível de ingestão } \\
\text { Intake level }\end{array}$} & & \multicolumn{3}{|c|}{$\begin{array}{c}\text { Temperatura }\left(13^{\circ} \mathrm{C}\right) \\
\text { Temperature }\end{array}$} \\
\hline & $\begin{array}{l}\mathrm{IR}^{2} \\
(\%)\end{array}$ & $\begin{array}{c}\text { Ingestão de EMAn } \\
\text { (kcal/kg0,75/dia) } \\
M E \text { intake } \\
\left(\text { kcal } \mathrm{kg}^{7}{ }^{75} / \text { day }\right)\end{array}$ & 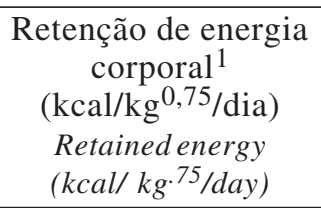 & $\begin{array}{c}\text { Produção de calor } \\
\text { (kcal/kg0,75/dia) } \\
\text { Heat production } \\
\text { (kcal/ } \mathrm{kg}^{75} / \text { day) }\end{array}$ \\
\hline $\mathrm{T} 1-$ ad libitum & 100,0 & 188,68 & 58,74 & 129,94 \\
\hline $\mathrm{T} 2-70 \%$ & 77,7 & 146,57 & 32,67 & 113,90 \\
\hline $\mathrm{T} 3-50 \%$ & 56,5 & 106,66 & 11,29 & 95,37 \\
\hline $\mathrm{T} 4-30 \%$ & 39,1 & 73,69 & $-12,47$ & 86,16 \\
\hline \multirow{2}{*}{$\begin{array}{l}\text { Nível de ingestão } \\
\text { Intake level }\end{array}$} & & \multicolumn{3}{|c|}{$\begin{array}{c}\text { Temperatura }\left(13^{\circ} \mathrm{C}\right) \\
\text { Temperature }\end{array}$} \\
\hline & $\begin{array}{l}\mathrm{IR}^{2} \\
(\%)\end{array}$ & $\begin{array}{c}\text { Ingestão de EMAn } \\
\text { (kcal/kg0,75/dia) } \\
\text { ME intake } \\
\left.\text { (kcal/ } \mathrm{kg}^{7} \cdot 75 / \text { day }\right)\end{array}$ & 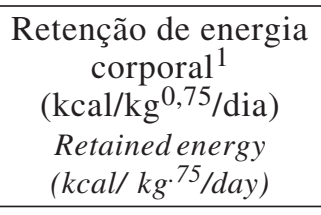 & $\begin{array}{c}\text { Produção de calor } \\
\text { (kcal/kg0,75/dia) } \\
\text { Heat production } \\
\text { (kcal/ } \mathrm{kg}^{75} / \text { day) }\end{array}$ \\
\hline $\mathrm{T} 1$ - ad libitum & 100,0 & 151,70 & 36,69 & 115,01 \\
\hline $\mathrm{T} 2-70 \%$ & 83,0 & 125,95 & 19,19 & 106,75 \\
\hline $\mathrm{T} 3-50 \%$ & 60,6 & 91,87 & 5,22 & 86,65 \\
\hline $\mathrm{T} 4-30 \%$ & 40,7 & 61,67 & $-17,40$ & 79,08 \\
\hline
\end{tabular}

R. Bras. Zootec., v.33, n.2, p.382-390, 2004 
produção de calor ( $\left.\mathrm{kcal} / \mathrm{kg}^{0,75} / \mathrm{dia}\right)$ para poedeiras Leghorn brancas, comparados aos obtidos com poedeiras comerciais semi-pesadas. A diferença em torno de $16,2 \mathrm{kcal} / \mathrm{kg}^{0,75} /$ dia decorreu, provavelmente, de diferentes taxas metabólicas das linhagens.

Existem diferentes fatores que interferem no metabolismo do animal como tamanho corporal, ciclo cicardiano, idade, nutrição, composição corporal, tamanho dos órgãos e do corpo, função exercida pelo animal e diferenças entre as espécies. Animais em fase de crescimento têm maior exigência de energia para mantença, tendo em vista que a síntese protéica é alta e os gastos com energia são extremamente elevados, aumentando a produção de calor (Blaxter, 1989)

$\mathrm{Na}$ Tabela 4, são apresentados os dados de ingestão de energia, energia retida e produção de calor das aves criadas em piso. Pode-se observar que as maiores exigências de EMm também foram obtidas para as aves submetidas a $13^{\circ} \mathrm{C}$.

As exigências de EMm das aves criadas em piso foram superiores às das aves mantidas em gaiolas em todas as temperaturas. Maiores gastos de energia com atividade muscular para locomoção e maior produção de calor das aves no piso auxiliam a explicação desta diferença (Tabela 5).

\begin{tabular}{|c|c|c|c|}
\hline \multicolumn{4}{|l|}{$\begin{aligned} \text { Tabela } 3 \text { - Equaçoes de } \\
\text { calor, em funç } \\
\text { e eficiência de } \\
\text { para mantenç } \\
\text { Regression equ } \\
\text { energy, MEm, } \\
(\mathrm{kg}) \text { and efficier }\end{aligned}$} \\
\hline \multirow{2}{*}{$\begin{array}{l}\text { Equação } \\
\text { Equation }\end{array}$} & $\mathrm{R}^{2}$ & Exigência $\left(\mathrm{kcal} / \mathrm{kg}^{0,75} / \mathrm{dia}\right)$ & Eficiência \\
\hline & & Requirement $(\mathrm{kcal} / \mathrm{kg} \cdot 75 /$ day $)$ & Efficiency \\
\hline \multicolumn{4}{|l|}{$13^{\circ} \mathrm{C}$} \\
\hline \multirow{2}{*}{$\begin{array}{c}\mathrm{ER}=-67,711+0,6091 . \mathrm{EMI} \\
\mathrm{PC}=77,83 \mathrm{e}^{0,0031 . \mathrm{EMI}}\end{array}$} & 0,98 & $\mathrm{EMm}=111,17$ & $k g=0,61$ \\
\hline & 0,96 & $\mathrm{ELm}=77,83$ & $k m=0,70$ \\
\hline \multicolumn{4}{|l|}{$21^{\circ} \mathrm{C}$} \\
\hline \multirow{2}{*}{$\begin{array}{c}\mathrm{ER}=-55,229+0,6035 . \mathrm{EMI} \\
\mathrm{PC}=65,20 \mathrm{e}^{0,0037 . \mathrm{EMI}}\end{array}$} & 0,97 & $\mathrm{EMm}=91,51$ & $k g=0,60$ \\
\hline & 0,94 & $E L m=65,20$ & $k m=0,71$ \\
\hline \multicolumn{4}{|l|}{$30^{\circ} \mathrm{C}$} \\
\hline \multirow{2}{*}{$\begin{array}{l}\mathrm{ER}=-50,085+0,566 . \mathrm{EMI} \\
\mathrm{PC}=59,19 \mathrm{e}^{0,0045} \cdot \mathrm{EMI}\end{array}$} & 0,93 & $\mathrm{EMm}=88,49$ & $k g=0,57$ \\
\hline & 0,91 & $\mathrm{ELm}=59,19$ & $k m=0,67$ \\
\hline
\end{tabular}

Tabela 4 - Ingestão de EMAn, energia corporal retida e produção de calor das aves criadas em piso $^{1}$

Table 4 - ME intake, retained energy and heat production of broiler breeder reared in floor

\begin{tabular}{|c|c|c|c|}
\hline \multirow[t]{2}{*}{$\begin{array}{l}\text { Parâmetros }\left(\mathrm{kcal} / \mathrm{kg}^{0,75} / \mathrm{dia}\right) \\
\text { Parameters }\left(\text { kcal } / \mathrm{kg}^{.75} / \text { day }\right)\end{array}$} & \multicolumn{3}{|c|}{$\begin{array}{c}\text { Temperatura }\left({ }^{\circ} \mathrm{C}\right) \\
\text { Temperature }\end{array}$} \\
\hline & 13 & 21 & 30 \\
\hline $\begin{array}{l}\text { Ingestão de EM } \\
\text { ME intake }\end{array}$ & 164,98 & 169,15 & 169,94 \\
\hline $\begin{array}{l}\text { Energia retida total } \\
\text { Total retained total energy }\end{array}$ & 20,80 & 33,97 & 33,38 \\
\hline $\begin{array}{l}\text { Produção de calor } \\
\text { Heat production }\end{array}$ & 144,18 & 135,18 & 136,56 \\
\hline $\begin{array}{l}\text { Exigência de EM para mantença } \\
M E \text { requirement for maintenance }\end{array}$ & 130,83 & 112,86 & 110,97 \\
\hline
\end{tabular}

1 Valores de composição total corporal (corpo+penas+ovos).

${ }^{1}$ Body composition (body+feathers+eggs). 
Os valores de eficiência de utilização da energia acima da mantença foram de 60,9, 60,4 e 56,6\% nas temperaturas de 13,21 e $30^{\circ} \mathrm{C}$, respectivamente. Valores próximos $(62,4 \%)$ foram encontrados por Reid et al. (1978) para poedeiras leves determinados em temperaturas que variaram de 21,3 a $26,5^{\circ} \mathrm{C}$. Basaglia (1999) também determinou eficiências semelhantes (66, 62 e 69\%), nas temperaturas de 12, 22 e $31^{\circ} \mathrm{C}$, respectivamente.

Observou-se pouca influência das temperaturas nas eficiências de utilização da energia da dieta para mantença, que foram de 70,71 e $67 \%$ para as temperatura de 13,21 e $30^{\circ} \mathrm{C}$, respectivamente. Em experimentos realizados no Brasil, utilizando a mesma metodologia e condições de temperatura, Silva (1999), Basaglia (1999) e Longo (2000) encontraram valores similares de 75, 76 e $72 \%$ para reprodutoras pesadas em crescimento, 72,71 e $74 \%$ para poedeiras comerciais adultas e 76, 80 e $76 \%$ para frangos de corte em crescimento.

Os valores de produção de calor diminuíram com a redução na ingestão de energia. Segundo Li et al. (1991), aves recebendo ração à vontade têm aumento na produção de calor durante a alimentação, o que pode ser atribuído, principalmente, ao ato da alimentação, e não ao trabalho de digestão (Van Kampen, 1976b) ou ao consumo alimentar (Blaxter, 1989).

As exigências de EMAm para aves mantidas em gaiolas foram de 111,$2 ; 91,5$ e $88,5 \mathrm{kcal} / \mathrm{kg}^{0,75} / \mathrm{dia}$, enquanto para aves criadas em piso foram de 130,8; 112,9 e $111,0 \mathrm{kcal} / \mathrm{kg}^{0,75} / \mathrm{dia}$, respectivamente para as temperaturas de 13,21 e $30^{\circ} \mathrm{C}$ (Tabela 5). Conseqüentemente, as aves sobre piso tiveram maior produção de calor: 144,18; 135,18 e 136,56kcal $/ \mathrm{kg}^{0,75} / \mathrm{dia}$ em piso e 77,$83 ; 65,20$ e $59,19 \mathrm{kcal} / \mathrm{kg}^{0,75} / \mathrm{dia}$ em gaiolas. Jonhson \& Farrell (1983) e Spratt et al. (1990) encontraram valores similares de EMm, quando alojaram reprodutoras pesadas em câmara de respiração à temperatura de $21^{\circ} \mathrm{C}, 87,24 \mathrm{e} 87,71 \mathrm{kcal} / \mathrm{kg}^{0,75} / \mathrm{dia}$. Da mesma forma, as exigências de EMm para aves criadas em piso foram superiores às das aves criadas em gaiolas, em média, $21 \mathrm{kcal} / \mathrm{kg}^{0,75} / \mathrm{dia}(21,80 \%)$.

Segundo Austic \& Nesheim (1990), as exigências de energia para atividades são estimadas em aproximadamente 50\% do metabolismo basal e são influenciadas pelas condições de alojamento. As demandas das aves alojadas em gaiolas diferem daquelas com aves criadas em piso pela restrição de atividade que resulta em menor perda de calor. Deighton \& Hutchinson (1940), citados por Van Kampen (1976c), verificaram que a perda de calor total aumentou $42 \%$ para aves que se encontravam em pé quando comparadas com as sentadas, enquanto Deshazer (1969) encontrou valores que variaram de 20 a $30 \%$ de aumento na perda de calor.

Em experimentos com poedeiras comerciais alojadas em câmaras de respiração, verificou-se que aproximadamente $20 \%$ da produção diária de calor são atribuídos à atividade (Macleod et al., 1982; Boshouwers \& Nicaise, 1983). Li et al. (1991) relatam que em torno de $19 \%$ da energia gasta por poedeiras comerciais com a produção de calor decorreu da atividade e, sob iluminação, a produção de calor foi 33\% superior àquelas submetidas em ambiente escuro, devido à menor taxa de metabolismo basal. Boshouwers \& Nicaise (1985), em experimento realizado com poedeiras (Shaver 288) com 62 semanas de idade, alojadas em calorímetro com temperatura de $21^{\circ} \mathrm{C}$, demonstraram que $25 \%$ da produção de calor foram ocasionados pela atividade física. Os autores encontraram produção total de calor de

\begin{tabular}{|c|c|c|c|}
\hline $\begin{array}{c}\text { Temperatura }\left({ }^{\circ} \mathrm{C}\right) \\
\text { Temperature }\end{array}$ & \multicolumn{3}{|c|}{$\begin{array}{l}\text { Exigência de EMAn para mantença }\left(\mathrm{kcal} / \mathrm{kg}^{0,75} / \mathrm{dia}\right) \\
\text { Energy requirements for maintenance }\left(\mathrm{kcal} / \mathrm{kg}^{0.75} / \text { day }\right)\end{array}$} \\
\hline & Gaiola & Piso & Diferenças \\
\hline & Cage & Floor & Differences \\
\hline 13 & 111,17 & 130,83 & $+19,66$ \\
\hline 21 & 91,51 & 112,86 & $+21,35$ \\
\hline 30 & 88,49 & 110,97 & $+22,48$ \\
\hline $\begin{array}{l}\text { Médias } \\
\text { Means }\end{array}$ & 97,06 & 118,22 & $+21,16$ \\
\hline
\end{tabular}

R. Bras. Zootec., v.33, n.2, p.382-390, 2004 
$110,7 \mathrm{kcal} / \mathrm{kg}^{0,75} \mathrm{com}$ o calor gasto para atividade física de $28 \mathrm{kcal} / \mathrm{kg}^{0,75}$. Esses autores verificaram também que a influência da luz na produção de calor foi maior durante o dia $\left(121 \mathrm{kcal} / \mathrm{kg}^{0,75} / \mathrm{dia}\right)$ que à noite $\left(88 \mathrm{kcal} / \mathrm{kg}^{0,75} / \mathrm{dia}\right)$. Segundo Balnave et al. (1978), as perdas de calor resultantes da atividade física são difíceis de estimar. Evidências indicam que a perda de calor pela atividade associada com alimentação e processos mecânicos da produção de ovos é pequena (Balnave, 1974; Van Kampen, 1976a). Entretanto, outros tipos de atividades têm efeitos variáveis na perda de calor e diferenças genéticas de temperamento, de plano de nutrição, grau de empenamento e temperatura ambiente são fatores importantes (Balnave, 1974).

As diferenças nas exigências de EMm entre as aves reprodutoras criadas em piso e gaiolas foram significativas neste estudo. Essa diferença é de grande valor, uma vez que a maioria dos sistemas de criação utilizados é em piso e os trabalhos realizados para determinação das exigências energéticas ocorrem freqüentemente em gaiolas. Dessa forma, erros no fornecimento de ração para matrizes pesadas sob condições comerciais podem estar relacionados a diferenças desta estimativa no dois sistemas e podem ajudar a explicar ganhos de peso inesperados com os programas de arraçoamento usados comercialmente.

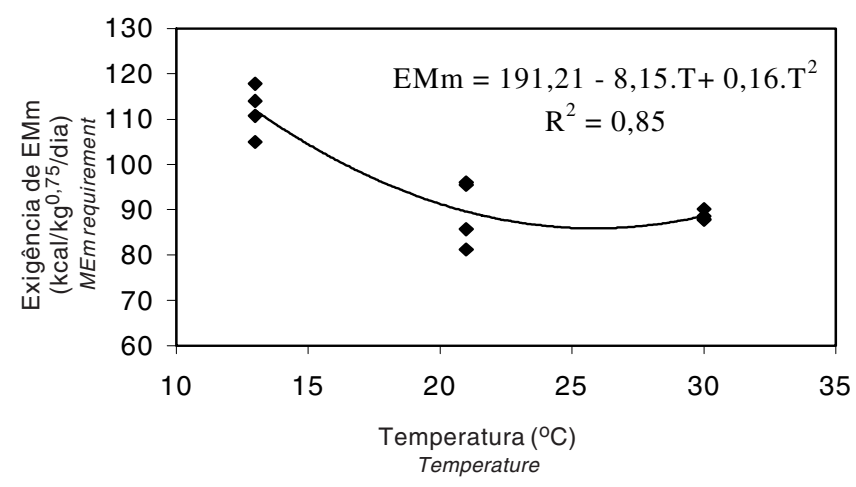

Figura 1 - Efeito da temperatura ambiente sobre as exigências de EMm das aves reprodutoras pesadas alojadas em gaiola.

Figure 1 - Effect of the environmental temperature on MEm of broiler breeder hens placed in cages.
Os efeitos da temperatura sobre as exigências de EMm são apresentados nas Figuras 1 e 2. Foram verificadas respostas quadráticas $(\mathrm{P}<0,05)$, tanto para as aves criadas em gaiolas como em piso, demonstrando que as exigências de energia para mantença diminuíram até aproximadamente $26^{\circ} \mathrm{C}$, voltando a aumentar a partir dessa temperatura. Este resultado difere de outros autores que relatam efeito linear da temperatura sobre as exigências de energia para mantença das aves (Combs, 1968; Hurwitz \& Bornstein, 1973; Emmans, 1974; Waldroup et al., 1976; Rostagno et al., 1983; Sakomura, 1989; NRC, 1994; Silva, 1995; Basaglia, 1999; Silva, 1999).

Os resultados obtidos neste trabalho demonstram que a temperatura exerce efeito sobre as exigências de aves, com pequenas diferenças na produção de calor, entre 19 e $27^{\circ} \mathrm{C}$, mas com necessidade de geração de calor para manter a temperatura em condições de temperaturas ambientais mais baixas. Já, com temperaturas acima de $27^{\circ} \mathrm{C}$, surgem demandas de energia para manter os mecanismos de resfriamento corporal. Entretanto, estes limites estabelecidos não são fixos, pois fatores como peso corporal e aumento no consumo alimentar, empenamento e na atividade promovem variações nas respostas das aves em face às mudanças nas condições ambientais (Leeson \& Summers, 1997).

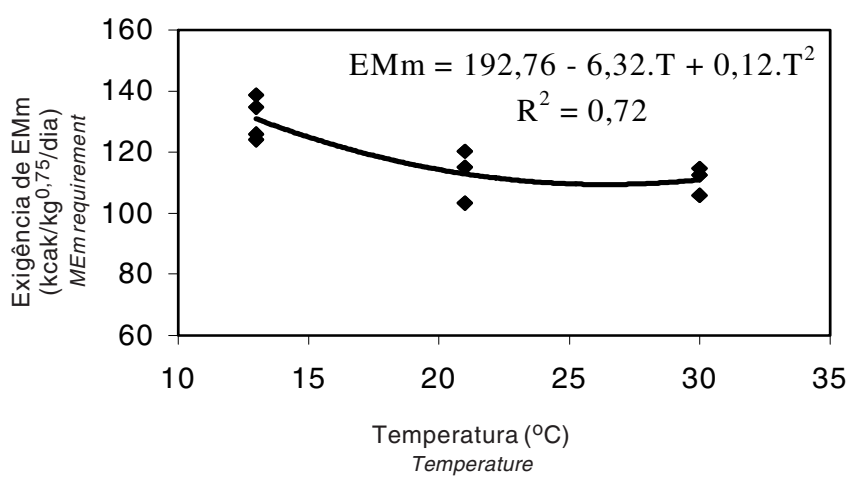

Figura 2 - Efeito da temperatura ambiente sobre as exigências de EMm das aves alojadas em piso.

Figure 2 - Effect of the environmental temperature on MEm of broiler breeder hens reared in floor.

R. Bras. Zootec., v.33, n.2, p.382-390, 2004 


\section{Conclusões}

O efeito da temperatura sobre as exigências de EMm resultou nos modelos $E M m=191,21-$ 8,15.T+0,16. $\mathrm{T}^{2}$ para as aves criadas em gaiolas e $\mathrm{EMm}=192,76-6,32 \cdot \mathrm{T}+0,12 \cdot \mathrm{T}^{2}$ para criadas em piso.

As reprodutoras pesadas criadas em piso tiveram exigências de EMm em média $21 \mathrm{kcal} / \mathrm{kg}^{0,75} /$ dia superior às exigências das aves mantidas em gaiolas.

De acordo com os resultados encontrados neste trabalho, sugere-se que os fatores temperatura e sistema de criação sejam levados em consideração no que se refere ao fornecimento de energia metabolizável para manteça de aves reprodutoras pesadas.

\section{Agradecimento}

À Fundação de Amparo à Pesquisa do Estado de São Paulo (FAPESP) e à Granja Rezende, pelo suporte financeiro.

\section{Literatura Citada}

ASSOCIATION OF AGRICULTURAL CHEMISTS - AOAC. Official methods of analysis. 16.ed. Washington, D.C.: 1990. 1094p.

AUSTIC, R.C.; NESHEIM, M.C. Poultry production. 13.ed. Philadelphia: Lea \& Febiger, 1990. 325p.

BALNAVE, D. Biological factors affecting energy expenditure. In. MORRIS, T.R.; FREMAN, B.M. (Eds.). Energy requirements of poultry (Poultry Science Symposium, 9). Edinburgh: British Poultry, 1974. p.25-46.

BALNAVE, D.; FARRELL, D.J.; CUMMING, R.B. The minimum metabolizable energy requirements of laying hens. World's Poultry Science Journal, v.34, n.1, p.149-154, 1978.

BASAGLIA, R. Equações de predição das exigências das exigências de energia e proteína para poedeiras leves. Jaboticabal: Universidade Estadual Paulista, 1999. 158p. Tese (Doutorado em Zootecnia) - Universidade Estadual Paulista, 1999.

BLAXTER, K. Energy metabolism in animal and man. Cambridge: 1989. 336p.

BOSHOUWERS, F.M.G.; NICAISE, E. The respiration quotient in indirect calorimetric measurements. British Poultry Science, v.24, n.1, p.273-279, 1983.

BOSHOUWERS, F.M.G.; NICAISE, E. Automatic gravimetric calorimeter with simultaneous recording of physical activity for poultry. British Poultry Science, v.26, n.1, p.534-541, 1985.

CHWALIBOG, A. Studies on energy metabolism in laying hens. Denmark: Institute of Animal Science, 1985. (Report, 578).

COMBS, G.F. Amino acid requirements of broilers and laying hens. In: MARYLAND NUTRITION CONFERENCE, 1968, Maryland. Proceedings... Maryland: 1968. p.86-96.
EMMANS, G.C. The effect of temperature o performance of laying hens. In: MORRIS, T.R.; FREEMAN, B.M. (Eds.) Energy requirements of poultry. Edinburgh: British Poultry Science, 1974. p.79-90.

FARREL, D.J. General principles and assumptions of calorimetry. In: MORRIS, T.R.; FREEMAN, B.M. (Eds.) Energy requirements of poultry. Edinburgh: British Poultry Science, 1974. p.1-23.

GRIMBERGEN, A. H. M. Energy expenditure under productive conditions. In: MORRIS, T.R.; FREEMAN, B.M. (Eds.). Energy requirements of poultry. Edinburgh: British Poultry Science, 1974. p.61-71.

HUBBARD FARMS. GUIA DE MANEJO :REPRODUTORAS HUBBARD CLÁSICA Y HI-Y. Hubbard Farms. New Hampshire, USA. 21 p. 2000.

HURWITZ, L.; BORNSTEIN, S. The protein and amino acid requirements of laying hens: suggested models for calculation. Poultry Science, v.52, n.3, p.1124-1134, 1973.

JOHNSON, R.J.; FARRELL, D.J. Energy metabolism of groups of broiler breeders in open-circuit respiration chambers. British Poultry Science, v.24, n.2, p.439-453, 1983.

LEESON, S.; SUMMERS, J.D. Commercial poultry nutrition. 2.ed. Guelph: 1997. 355p.

LI, Y.; ITO, T.; YANAMOTO, S. Diurnal variation of heat production related to some physical activities in laying hens. British Poultry Science, v.32, n.4, p.821-827, 1991.

LOFGREEN, G.P.; GARRETT, W.N. A system for expressing net energy requirements and feed values for growing and finishing beef cattle. Journal of Animal Science, v.28, p.693-704, 1968.

LONGO, F.A. Estudo do metabolismo energético e do crescimento em frangos de corte. Jaboticabal: Universidade Estadual Paulista, 2000. 76p. Dissertação (Mestrado em Zootecnia) - Universidade Estadual Paulista, 2000.

MACLEOD, M.G.; JEWITT, T.R.; WHITE, J. et al. The contribution of locomotion activity to energy expenditure in the domestic fowl. In: EKERN, A.; SUNDSTOL, S. (Eds.) Energy metabolism of farm animal. Norway: The Agricultural University of Norway, 1982. p.297-300.

MACLEOD, M.G.; JEWITT, T.R. Maintenance energy requirements of laying hens? A comparison of measurements made by two methods based on indirect calorimetry. British Poultry Science, v.29, n.1, p.63-74, 1988.

MARSDEN, A.; MORRIS, T.R. Quantitative review of the effects of environmental temperature on food intake, egg output and energy balance in laying pullets. British Poultry Science, v.32, n.4, p.821-827, 1987.

NATIONAL RESEARCH COUNCIL - NRC. Committee on Animal Nutrition. Subcommittee on Poultry Nutrition. Nutrient requirements of poultry. 9.ed. Washington: National Academy of Sciences, 1994. 155p.

PEGURI, A.; COON, C.N. Development and evaluation of prediction equations for metabolizable energy and true metabolizable energy intake for Dekalb XL-Link White Leghorn hen. In: MINNESOTA NUTRITION CONFERENCE AND DEGUSSA TECHNOLOGY SYMPOSIUM, 49., 1988, Bloming. Proceedings ... Bloming: 1988. p.199-211.

RABELLO, C.B.V. Equações de predição das exigências de energia e proteína para reprodutoras pesadas na fase de produção. Jaboticabal: Universidade Estadual Paulista, 2001. 121p. Tese (Doutorado em Zootecnia) - Universidade Estadual Paulista, 2001.

REID, B.L.; VALENCIA, M.E.; MAIORINO, P.M. Energy

R. Bras. Zootec., v.33, n.2, p.382-390, 2004 
utilization of laying hens. I. Energetic efficiencies of maintenance and production. Poultry Science, v.57, n.2, p.461-465, 1978.

ROSTAGNO, H.S.; SILVA, D.J.; COSTA, P.M.A. et al. Composição de alimentos e exigências nutricionais de aves e suínos (Tabelas Brasileiras). Viçosa, MG: Universidade Federal de Viçosa, 1983. 59p.

SAKOMURA, N.K. Exigências nutricionais de energia metabolizável para reprodutoras pesadas, poedeiras semipesadas e leves. Viçosa, MG: Universidade Federal de Viçosa, 1989. 242p. Tese (Doutorado em Zootecnia) - Universidade Federal de Viçosa, 1989.

STATISTICAL ANALYSES SYSTEM - SAS. SAS guide: statistics. Version 6. 12.ed. Cary: 1996.

SILVA, R. Exigências de energia metabolizável para frangas de postura de 1 e 18 semanas de idade. Jaboticabal: Universidade Estadual Paulista, 1995. 76p. Dissertação (Mestrado em Zootecnia) - Universidade Estadual Paulista, 1995.

SILVA, R. Equações de predição das exigências das exigências de energia e proteína para matrizes pesadas em crescimento. Jaboticabal: Universidade Estadual Paulista, 1999. 153p. Tese (Doutorado em Zootecnia) - Universidade Estadual Paulista, 1999.
SPRATT, R.S.; BAYLEY, H.S.; McBRIDE, B.W. et al. Energy metabolism of broiler breeder hens. 1. The partition of dietary energy intake. Poultry Science, v.65, n.8, p.13391347, 1990.

VAN KAMPEN, M. Activity and energy expenditure in laying hens: 1 . The energy cost of nesting activity and oviposition. Journal Agriculture Science, v.86, n.2, p.471-473, 1976a.

Van KAMPEN, M. Activity and energy expenditure in laying hens: 2. The energy cost of exercise. Journal Agriculture Science, v. 87, n.1, p.81-84, 1976 b.

Van KAMPEN, M. Activity and energy expenditure in laying hens: 3 . The energy cost of eating and posture. Journal of Agriculture Science, v.87, n.1, p.85-88, 1976c.

WALDROUP, P.W.; HAZEN, K.R.; BUSSEL, W.D. et al. Studies on the daily protein and amino acid needs of broiler breeders hens. Poultry Science, v.55, n.9, p.2342-2347, 1976.

Recebido em: 26/07/02

Aceito em: 09/09/03 